\section{$\epsilon_{\mathrm{s}}$}

- Additional material is

published online only. To view please visit the journal online (http://dx.doi.org/10.1136/ thoraxjnl-2016-208939).

\section{'Department of Pulmonary} Medicine, University of

Amsterdam, Academic Medical Center (AMC), Amsterdam, The Netherlands

${ }^{2}$ Department of Pulmonary Medicine, Free University Medical Center (VUMC) Amsterdam, The Netherlands ${ }^{3}$ Department of Radiology, University of Amsterdam, Academic Medical Center (AMC), Amsterdam, The Netherlands

${ }^{4}$ Department of Pathology and Cardiac Surgery, Free University Medical Center (VUMC),

ICaR-VU, Amsterdam, The Netherlands

${ }^{5}$ Department of Pathology, University of Amsterdam, Academic Medical Center, Amsterdam, The Netherlands

Correspondence to Dr Peter I Bonta, Department of Pulmonary Medicine, University of Amsterdam, Academic Medical Center (AMC) Meibergdreef 9, Amsterdam 1105AZ, The Netherlands: p.i.bonta@amc.nl

Received 15 June 2016 Revised 30 August 2016 Accepted 5 September 2016 Published Online First 28 September 2016

\title{
Pulmonary endarterectomy for calcified amorphous tumour-related pulmonary hypertension
}

\author{
Peter I Bonta, ${ }_{1}^{1}$ Harm J Bogaard ${ }_{1}{ }^{2}$ Inge van den Berk, ${ }^{3}$ Hans W M Niessen, ${ }^{4}$ \\ Allard van der Wal, ${ }^{5}$ Petr Symersky ${ }^{4}$
}

A 42-year-old patient presenting with exertional dyspnoea was diagnosed with functional class II pulmonary hypertension $(\mathrm{PH})$ with severe tricuspid valve insufficiency due to cardiac and pulmonary artery obstruction by calcified amorphous tumour (paCAT). Bilateral (sub)segmental perfusion defects were seen on ventilation/perfusion scan (see online supplementary figure S1), while the CT angiogram showed large amounts of calcified radio-opaque material in absence of radiological characteristics of chronic thromboembolic pulmonary hypertension (CTEPH), such as webs, stenosis or pouches (figure 1A, C). After multi-institutional (Amsterdam, NL and Papworth, Cambridge, UK) assessment of the case, a decision was made to perform a complete, bilateral, pulmonary endarterectomy (PEA) combined with tricuspid valve replacement. During PEA bone-hard material was retrieved that lacked the (sub)segmental pulmonary artery tails seen in CTEPH (figure $2 \mathrm{~A}$ and $\mathrm{B}$ ). After PEA minimal residual paCAT was detected on follow-up CT angiogram (figure 1B,D). Figure 2 aligns paCAT in the left main pulmonary artery on CT angiogram (figure 1A, red box) down to macroscopy and histopathological microscopy. The macroscopy images show the complete PEA specimen (figure 2A) and upright-positioned left main paCAT specimen that illustrates the bone-hard nature of the tissue (figure 2B). Histopathological images show, next to the pulmonary artery media at the dissection level (figure 2C, arrow heads), large amounts of nodular calcific deposits (figure $2 \mathrm{C}, \mathrm{D}^{*}$ ) in a fibrous matrix. Focally, calcified amorphous material is surrounded by haemosiderin-laden macrophages (figure 2D, arrows). These image features prove this case a paCAT. After PEA mean pulmonary artery pressure fell from 31 to $15 \mathrm{~mm} \mathrm{Hg}$ and pulmonary vascular resistance was reduced from 301 to 82 dynes $\mathrm{s} / \mathrm{cm}^{5}$. The patient's functional class improved from II to I and $6 \mathrm{~min}$ walking distance increased from 462 to $558 \mathrm{~m}$. No recurrence of disease occurred over $>2$ years of follow-up.
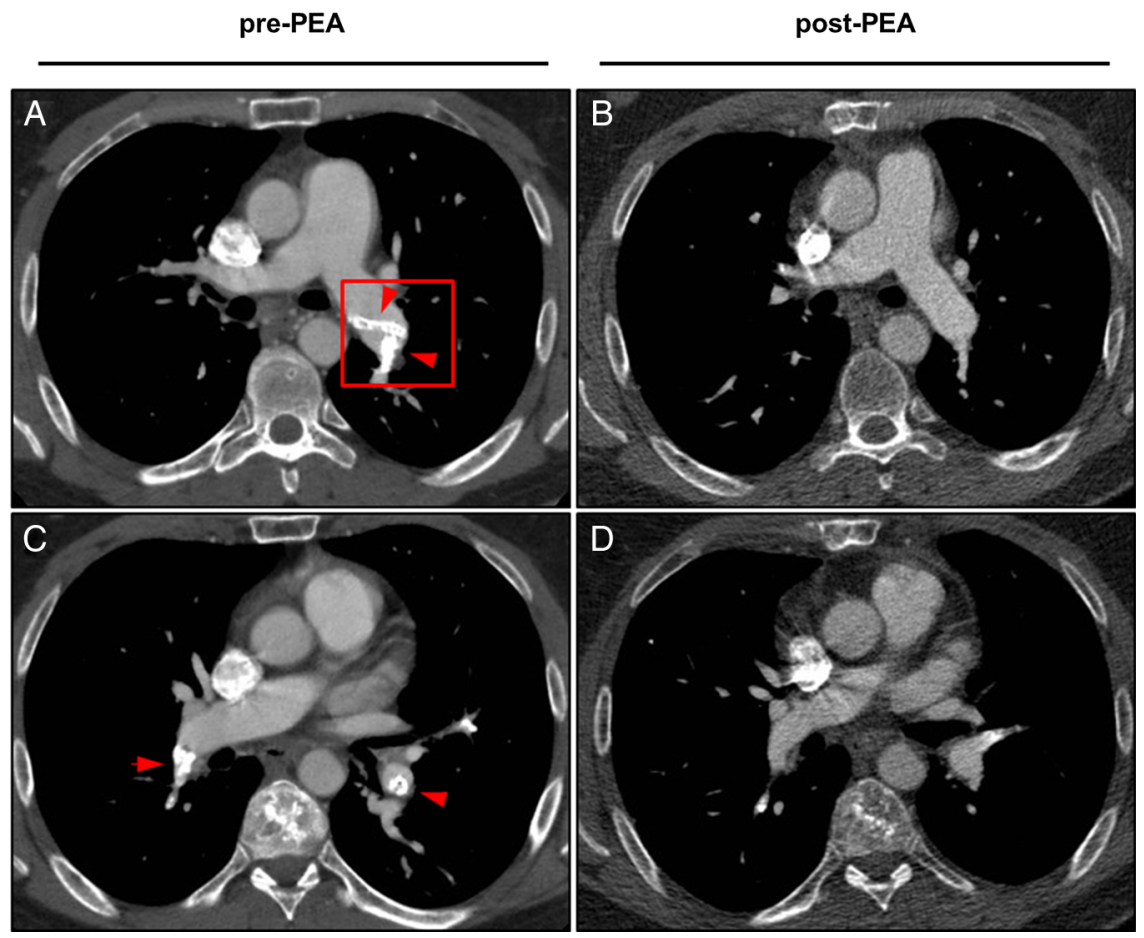

Figure 1 Axial sections of CT angiograms pre-PEA and post-PEA (A-D). Pre-PEA large amounts of calcified material (arrow heads) are present in the left main pulmonary artery $(A)$ and right segmental and left lower lobe pulmonary arteries (C). After PEA, near complete resolution of pulmonary artery obstruction by calcified amorphous tumour is seen (B and D). For additional pulmonary artery and cardiac CT sections pre-PEA and post-PEA (see online supplementary figures S2 and S3, respectively). PEA, pulmonary endarterectomy. 


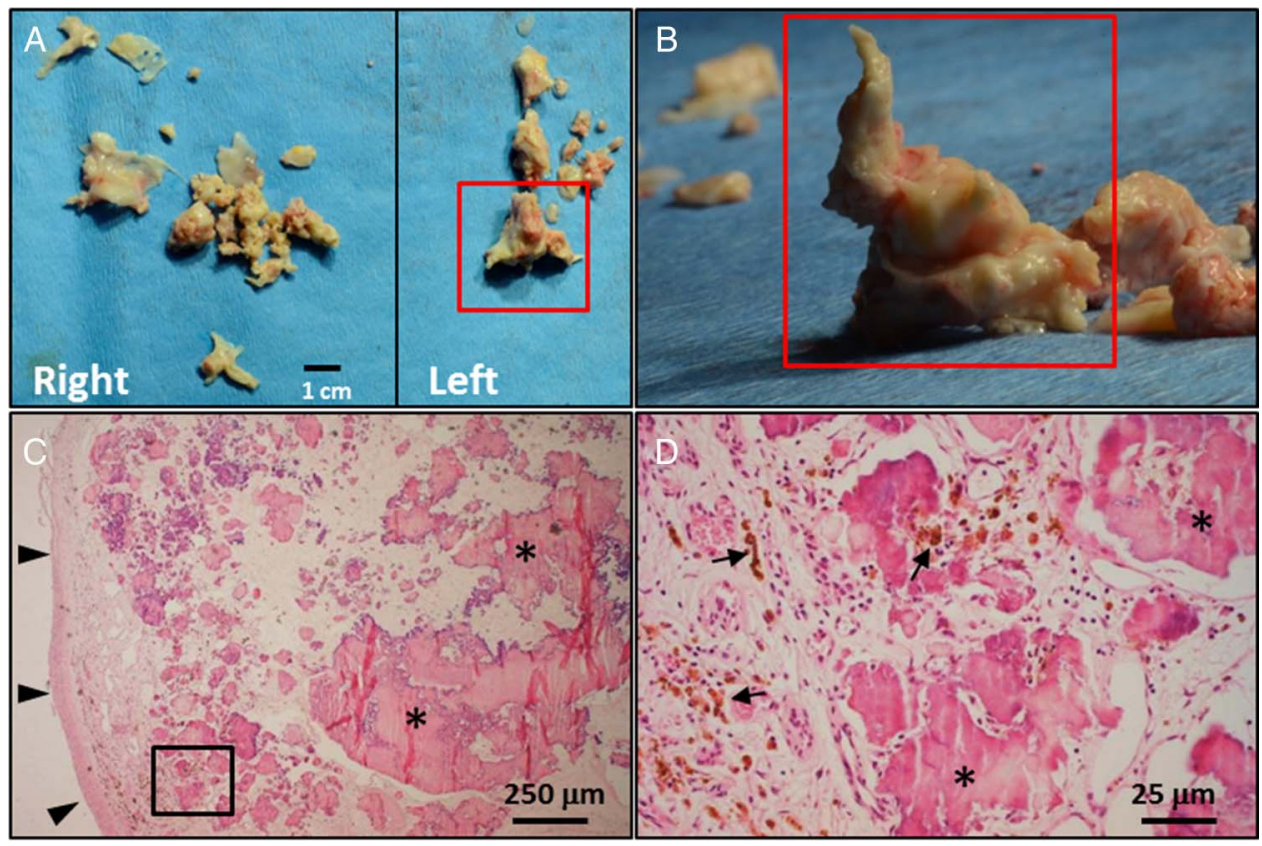

Figure 2 Macroscopic and histopathological microscopic images of pulmonary endarterectomy specimen (A-D). Left main pulmonary artery obstruction by calcified amorphous tumour on CT angiogram (Figure 1A, red box) is traced down to macroscopy (A and B, red boxes, photographs) and microscopy ( $C$ and $D, H \& E$ staining). Pulmonary artery media dissection level ( $C$, arrow heads); nodular calcific deposits in a fibrous matrix $\left(C\right.$ and $\left.D,{ }^{*}\right)$ and haemosiderin-laden macrophages $(D$, arrows $)$.

\section{DISCUSSION}

First classified as non-neoplastic intra-cardiac masses in $1997^{1}$ CATs can arise in the heart and are very rarely described in the pulmonary artery. ${ }^{2}{ }^{3}$ Lesions are characterised by nodular calcific deposits in a fibrous matrix background. ${ }^{1} 2$ The pathogenesis of CATs is unknown, but can be categorised as non-malignant since metastases have not been described. ${ }^{2}$ To our knowledge this is the first patient reported to have resolution of PH after complete, bilateral PEA for paCAT.

Contributors PIB, selected the case, wrote, edited, reviewed and submitted the paper; HJB, selected the case, edited and reviewed the manuscript; IvdB, reviewed and selected radiology images and reviewed the manuscript; HWMN, reviewed the pathology images and the manuscript; AvdW, reviewed and selected the pathology images and reviewed the manuscript; PS, selected the case, edited and reviewed the manuscript.

Competing interests None declared.

Patient consent Obtained.

Provenance and peer review Not commissioned; externally peer reviewed.

\section{REFERENCES}

1 Reynolds C, Tazelaar HD, Edwards WD. Calcified amorphous tumor of the heart (cardiac CAT). Hum Pathol 1997;28:601-6.

2 Hussain N, Rahman N, Rehman A. Calcified amorphous tumors (CATs) of the heart. Cardiovasc Pathol 2014:23:369-71.

3 Prifti E, Kajo E, Krakulli K, et al. Surgical excision of a giant calcified amorphous tumour of the right ventricle and right pulmonary artery. Interact Cardiovasc Thorac Surg 2015;21:805-7. 